\section{Hypertrophic Cranial Pachymeningitis with Inflammatory Valvular Disease}

To the Editor: We read with interest the article by Tojo et al (1) indicating an autopsy case of Wegener's granulomatosis associated with pachymeningitis. We describe a rare case of hypertrophic cranial pachymeningitis (HCP) presenting with valvular disease of the heart and a complete atrioventricular (AV) block.

A 47-year-old man with a 4-year history of HCP was admitted to our hospital because of exertional dyspnea. Four years earlier, the patient had been diagnosed with HCP by a head magnetic resonance imaging (MRI) and had received two courses of steroid therapy (methylprednisolone $500 \mathrm{mg}$ /day for 3 days) with resolution of the cranial nerve paralysis. The next year, he began to complain of dyspnea on exertion. On admission, he was febrile $\left(37.2^{\circ} \mathrm{C}\right)$. His lungs were clear, but pansystolic murmurs were heard over the apex. He had neither arthritis nor tonsillitis. A neurological examination showed oculomotor palsy in the right eye and a left cranial nerve XII palsy. The erythrocyte sedimentation rate was $65 \mathrm{~mm} / \mathrm{h}$, and the C-reactive protein titer was $7.7 \mathrm{mg} /$ dl. His serum immunoglobulin $G$ and $M$ were $2,203 \mathrm{mg} / \mathrm{dl}$ and $352 \mathrm{mg} / \mathrm{dl}$, respectively. Rheumatoid factor, antinuclear antibody, antistreptolysin $\mathrm{O}$ and serum angiotensin converting enzyme were all normal. A sagittal T1-weighted MRI showed an isointense lesion involving the upper cervical cord. An electrocardiogram showed a complete A-V block which had not been detected two years earlier. A subsequent echocardiographic examination revealed diffuse thickening of both mitral and aortic valves, with the absence of calcification and verrucae. Cardioangiography demonstrated both aortic and mitral valve regurgitation. An aortic valve replacement, a mitral valvuloplasty, and the implantation of a permanent pacemaker were performed. The removed aortic valve was soft and thick, and microscopic examination revealed that the aortic valve had been infiltrated with lymphocytes and plasma cells, but without granulation or vasculitis; this is consistent with acute and chronic inflammation. However, bacteriological and fungal cultures were negative. After the operation, his clinical course was uneventful and he was subsequently discharged.

$\mathrm{HCP}$ is a rare disease characterized by thickening of the dura mater, which leads to cranial nerve palsy, confusion and/or cerebellar ataxia (2). The pathogenesis of HCP is unclear, but in some studies it has been suggested to be related to autoimmune and connective disorders $(3,4)$. The histological findings of the affected dura in patients with HCP generally reveal acute and chronic inflammatory changes $(2,3)$, which was similar to the resected aortic valve in our patient. This may suggest a relationship between the HCP and the heart disease in our case, although no evidence indicating an underlying autoimmune process was noted. There has only been one other reported case of $\mathrm{HCP}$ associated with a complete A-V block (5). In both cases, the underlying etiologic mechanisms remain obscure. However, to our knowledge, this is the first case report of HCP associated with valvular disease.

\section{Ken-ichiro Inoue, Yohei Oda, Kiichiro Tomiyasu and Motoharu KonDO}

The First Department of Internal Medicine, Kyoto Prefectural University of Medicine, Kyoto

Reprint requests should be addressed to Ken-ichiro Inoue, the First Department of Internal Medicine, Kyoto Prefectural University of Medicine, Kyoto 6028566

\section{References}

1) Tojo J, Nishimaki T, Ohyanagi H, et al. An autopsy case of Wegener's granulomatosis with pachymeningitis. Intern Med 37: 711-715, 1998.

2) Mamelak AN, Kelly WM, Davis RL, Rosenblum ML. Idiopathic hypertrophic cranial pachymeningitis. Report of three cases. J Neurosurg 79: 270-276, 1993 (see comments).

3) Adler JR, Sheridan W, Kosek J, Linder S. Pachymeningitis associated with a pulmonary nodule. Neurosurgery 29: 283-287, 1991.

4) Kitano A, Shimomura T, Okada A, Takahashi K. Multifocal fibrosclerosis with intracranial pachymeningitis. Intern Med 34: 267-271, 1995.

5) Tanaka M, Suda M, Ishikawa $Y$, Fujitake J, Fujii H, Tatsuoka $Y$. Idiopathic hypertrophic cranial pachymeningitis associated with hydrocephalus and myocarditis: remarkable steroid-induced remission of hypertrophic dura mater. Neurology 46: 554-556, 1996. 